\title{
Estudo da qualidade dos Dados do Painel COVID-19 para crianças, adolescente e jovens, Espírito Santo - Brasil, 2020
}

\author{
A study assessing the quality of the COVID-19 Panel Data for children, adolescents and young individuals, \\ Espírito Santo - Brazil, 2020 \\ Estudio de calidad de datos del Panel COVID-19 para niños, adolescentes y jóvenes, Espírito Santo - \\ Brasil, 2020
}

Ethel Leonor Maciel ${ }^{1}$ (1)

Pablo Medeiros Jabor ${ }^{2}$ (C)

Etereldes Goncalves $\mathrm{Jr}^{3}$ (1)

Priscila Carminati Siqueira ${ }^{1}$ (B)

Thiago Nascimento do Prado ${ }^{1}$ (D)

Eliana Zandonade ${ }^{1}$ (1)

1. Universidade Federal de Espírito Santo, Programa de Pós-Graduação em Saúde Coletiva. Vitória, ES, Brasil.

2. Instituto Jones dos Santos Neves. Vitória, ES, Brasil.

3. Universidade Federal de Espírito Santo, Centro de Ciências Exatas. Vitória, ES, Brasil.

Autor correspondente:

Ethel Leonor Maciel.

E-mail: ethel.maciel@gmail.com.

Recebido em 18/12/2020.

Aprovado em 01/05/2021.

DOI:https://doi.org/10.1590/2177-9465-EAN-2020-0509

\section{Resumo}

Objetivo: descrever a completude dos dados e avaliar a qualidade do Banco de dados do Painel COVID-19 no Espírito Santo em 2020, quanto à completude de suas variáveis, bem como analisar a confirmação da doença e sua evolução por crianças, adolescentes e jovens. Métodos: estudo descritivo exploratório. A completude no preenchimento da ficha no Painel COVID-19 foi classificada como excelente (menos de $5 \%$ de preenchimento incompleto), bom ( $5 \%$ a $10 \%$ ), regular ( $10 \%$ a $20 \%$ ), ruim ( $20 \%$ a $50 \%$ ) ou muito ruim (50\% ou mais). Resultados: observou-se qualidade regular para o critério de confirmação (16\%), ruim para a classificação da doença (44\%) e status de notificação (30\%) e muito ruim para a evolução (79\%). Quanto às variáveis epidemiológicas, destaca-se a variável raça/cor da pele com completude regular (17\%). Conclusão e implicações para a prática: é necessário educação permanente dos profissionais para o preenchimento dos dados de forma correta. Tratando-se de uma pandemia por um vírus novo, esses dados devem estar disponíveis imediatamente, e com qualidade para que medidas de controle possam ser adotadas.

Palavras-Chave: COVID-19; Pandemia; Epidemiologia; Sistemas de Informação em Saúde; Notificação de Doenças.

\section{Abstract}

Objective: to describe the completeness of the data and evaluate the quality of the COVID-19 Panel Database in Espírito Santo in 2020, as to the completeness of its variables, as well as to analyze the confirmation of the disease and its evolution by children adolescents and young people. Methods: exploratory descriptive study. Completeness of filling in the form on the COVID-19 Panel was classified as excellent (less than $5 \%$ incomplete), good (5\% to $10 \%)$, fair $(10 \%$ to $20 \%)$, poor $(20 \%$ to $50 \%)$ or very bad ( $50 \%$ or more). Results: regular quality was observed for the confirmation criterion (16\%), poor for the classification of the disease $(44 \%)$ and notification status (30\%) and very poor for the evolution $(79 \%)$. Regarding the epidemiological variables, the race-skin color variable with regular completeness (17\%) stands out. Conclusion and implications for the practice: permanent education of professionals is necessary to fill in the data correctly. in the case of a pandemic due to a new virus, these data must be available immediately, and with quality so that control measures can be adopted.

Keywords: Coronavirus Infections; Pandemics; Epidemiology; Health Information Systems; Disease Notification.

\section{Resumen}

Objetivo: describir la exhaustividad de los datos y evaluar la calidad de la Base de Datos Panel COVID-19 en Espírito Santo en 2020 , en cuanto a la exhaustividad de sus variables, así como analizar la confirmación de la enfermedad y su evolución en niños, adolescentes y jóvenes. Métodos: estudio descriptivo exploratorio. La exhaustividad al completar el formulario en el Panel COVID-19 se clasificó como excelente (menos del 5\% incompleto), buena (5\% a 10\%), regular (10\% a $20 \%$ ), deficiente ( $20 \%$ a $50 \%$ ) o muy mala (50\% o más). Resultados: se observó calidad regular para el criterio de confirmación (16\%), mala para la clasificación de la enfermedad (44\%) y estado de notificación (30\%) y muy mala para la evolución (79\%). En cuanto a las variables epidemiológicas, se destaca la variable raza-color de piel con exhaustividad regular (17\%). Conclusión e implicaciones para la práctica: es necesaria la formación permanente de los profesionales para completar correctamente los datos. En el caso de una pandemia por un nuevo virus, estos datos deben estar disponibles de manera inmediata y con calidad para que se puedan adoptar medidas de control.

Palabras clave: Infecciones por Coronavirus; Pandemias; Epidemiología; Sistemas de Información en Salud; Notificación de Enfermedad. 


\section{INTRODUÇÃO}

O novo coronavírus identificado em Wuhan no fim de 2019, é uma doença de notificação compulsória imediata, que deve ser informada ao Ministério da Saúde em até 24 horas após o atendimento de um caso suspeito ou confirmado ${ }^{1}$. Para analisar e compreender os casos notificados por COVID-19, é necessário poder contar com um sistema de informações de qualidade, confiável, apresentar os campos preenchidos de forma correta, com dados completos e atualizados ${ }^{2}$. Não bastando para isso a informação apenas dos dados, mas a observação e demonstração sobre diferenças e desigualdades sociais que podem influir em desfechos, como cura e óbito. A baixa qualidade do preenchimento dos dados na ficha de notificação do COVID-19 limita a análise epidemiológica do agravo e dificulta o acompanhamento da dinâmica da doença, comprometendo a adoção de medidas de intervenção de forma adequada para minimizar os efeitos da doença e sua expansão para outras regiões ${ }^{3}$.

A completude se refere ao registro de todas as informações pertinentes à inclusão dos casos nos sistemas de informações de Saúde (SIS). Através desse indicador, é possível avaliar a qualidade das notificações de um determinado agravo ${ }^{4}$. Uma medida direta e fácil para avaliar a qualidade dos dados consiste em examinar a porcentagem de respostas "desconhecidas" ou em "branco" para itens presentes nos formulários da vigilância epidemiológica, uma vez que reflete a integralidade e a validade dos dados registrados ${ }^{2,3}$. Pensando em um país continental como o Brasil, as diferenças regionais são fundamentais para a organização de recursos e insumos para ação integrada e efetiva de acordo com os princípios do Sistema Único de Saúde (SUS). As informações em saúde são, portanto, indispensáveis, em momentos de pandemias, onde a exigência de resposta rápida é primordial para a decisão política, e, por conseguinte, a disponibilização dos dados, bem como a sua análise de qualidade se fazem necessárias ${ }^{5}$.

Durante a pandemia da COVID-19, um arranjo que até então, nunca havia sido feito no país, começou a ser colocado em ação, qual seja, a disponibilidade dos dados em painéis online. Essa disponibilidade para além da transparência movimenta um conjunto de pesquisadores que se dedicam a analisar e informar com base nos dados reportados sobre as infecções por coronavirus.

Sistemas com alta qualidade dos dados permitiriam identificar o perfil de crianças e jovens mais propensos à contaminação pelo SARS-CoV-2, e auxiliar na definição de um momento mais seguro para o retorno das aulas presenciais nas escolas de ensino fundamental e médio a partir dos dados epidemiológicos clínicos da COVID-19 na comunidade e principalmente, entre os escolares. Nesse sentido, o presente estudo teve por objetivo descrever a completude dos dados e avaliar a qualidade do Banco de dados do Painel COVID no Espírito Santo em 2020, quanto à completude de suas variáveis, bem como analisar a confirmação da doença e sua evolução por crianças, adolescentes e jovens.

\section{MÉTODOS}

Foi realizado um estudo descritivo exploratório da completude e da qualidade do banco de dados do Painel COVID, disponibilizado no sitio eletrônico: https://coronavirus.es.gov.br/painel-covid-19es, da Secretaria de Estado da Saúde do Espírito Santo (SESA) ${ }^{6}$

O banco de dados possui 320.870 casos notificados por COVID-19, no período de 17 de fevereiro até 20 de agosto de 2020. Foram analisados todos os dados de casos suspeitos de COVID-19, do painel, com idades de 0 a 19 anos, totalizando 27.351 registros.

O banco de dados do Painel COVID contém 41 variáveis, foram selecionadas para o estudo:

- Variáveis de confirmação da doença e evolução: Classificação (confirmados, descartados e suspeitos), Evolução (cura ou óbito por covid-19), Critério de Confirmação (clínico, clínico epidemiológico e laboratorial), Status da Notificação (em aberto e encerrado) ${ }^{7}$.

- Variáveis sociodemográficas: Bairro, Faixa Etária (0 a 4 anos, 5 a 9 anos, 10 a 14 anos e 15 a 19 anos), Sexo (masculino, feminino e indeterminado), Raça/Cor (amarela, branca, Indígena, parda, preta e ignorado), Escolaridade (Analfabeto, $1^{\text {a }}$ a $4^{\text {a }}$ série incompleta do EF, 4⿳亠丷a série completa do EF, 5 a 8 ${ }^{\text {a }}$ série incompleta do EF, Ensino fundamental completo, Ensino médio incompleto, Ensino médio completo, Educação superior incompleta, Educação superior completa, Não se aplica e Ignorado)

- Variáveis de sintomas, com respostas sim ou não: Febre, Dificuldade Respiratória, Tosse, Coriza, Dor de Garganta, Diarreia e Cefaleia.

- Variáveis de Comorbidades, com respostas sim ou não: Comorbidade de Pulmão, Comorbidade Cardiovascular, Comorbidade Renal, Comorbidade Diabetes, Comorbidade Tabagismo, Comorbidade Obesidade.

- Outras variáveis, com respostas "sim" ou "não": Ficou Internado, Viagem Brasil, Viagem Internacional, Profissional de Saúde, Possui Deficiência, Morador de Rua.

Os dados foram analisados de acordo com os critérios de avaliação da qualidade de dados propostos por Romero e Cunha ${ }^{8}$, e adaptados pela Comissão Econômica para a América Latina e Caribe (CEPAL) ${ }^{9}$. Para a análise desse artigo, foi utilizado o indicador não completude das variáveis, proposta na CEPAL, com dados do painel COVID-19.

O escore utilizado para as taxas de incompletude possui os seguintes graus de avaliação: "excelente", quando a variável apresenta menos de $5 \%$ de preenchimento incompleto, "bom" (5\% a $10 \%)$, "regular" (10\% a $20 \%)$, "ruim" (20\% a $50 \%)$ e "muito ruim" $(50 \% \text { ou mais) })^{8-10}$.

Considerou-se como categoria de dados faltantes, para a variável classificação, a categoria "suspeito", que indica a não investigação do caso. Para todas as demais variáveis, considerou- 
se como faltantes as categorias "em branco", "ignorado" e "não informado".

Foram realizadas frequências absolutas e percentuais, com associação entre as variáveis. Foi utilizado o programa SPSS, versão 20.0. Foram realizados testes qui-quadrado de associação. O nível de significância adotado foi de $5 \%$.

O presente estudo foi apreciado pelo Comitê de Ética em Pesquisa do Centro de Ciências da Saúde da Universidade Federal do Espírito Santo (CEP/CCS/UFES) e aprovado sob o parecer $n=3.908 .434$ de 20/05/2020.

\section{RESULTADOS}

Foram avaliados 27.351 registros de crianças, adolescentes e jovens de 0 a 19 anos no Espírito Santo. Encontrou-se $7.153(26,2 \%)$ na faixa etária de 0 a 4 anos, $4.298(15,7 \%)$ na faixa etária de 5 a 9 anos, $4.697(17,2 \%)$ na faixa etária de 10 a 14 anos e $11.203(41,0 \%)$ na faixa de 15 a 19 anos.

A variável "classificação dos pacientes", que informa o quanto estes pacientes foram investigados pela vigilância epidemiológica aponta $6099(22,3 \%)$ confirmados, $9190(33,6 \%)$ descartados e $12062(44,1 \%)$ casos suspeitos.

A Tabela 1 apresenta os resultados das categorias com falta de informação, com os percentuais e a classificação de qualidade, segundo Romero e Cunha. Observa-se que para a variável classificação, a categoria "suspeito" é a que se refere à incompletude da mesma, enquanto para as demais as categorias faltantes são "em branco", "ignorado" e "não informado". Entre as variáveis de confirmação da doença e evolução observa-se qualidade regular para o critério de confirmação (16\%), ruim para a classificação (44\%) e status de notificação (30\%) e muito ruim para a evolução (79\%). Quanto às variáveis sociodemográficas, destacamos a raça/cor com completude regular (17\%) e escolaridade como ruim (25\%). Ainda na Tabela 1 , no grupo de variáveis de sintomas e comorbidades, ambos tiveram todas as variáveis classificadas como completude excelente. Observa-se que estas variáveis já se encontram preenchidas no formulário eletrônico do ESUS (Estratégia de informatização do Sistema Único de Saúde) com a opção "Não". Quanto às demais variáveis, como "ficou internado" e viagens nacionais e internacionais foram classificadas de completude ruim.

A Tabela 2 abaixo apresenta a correlação de três variáveis, a Classificação, a Evolução e o Critério de Confirmação do diagnóstico. São apresentados os percentuais por linha e observase grande número de casos com a evolução em branco, bem como a grande quantidade de casos suspeitos, principalmente com evolução em branco e sem informação para o critério de confirmação.

Outra informação importante é quanto à situação de notificação (status da notificação). A Tabela 3 apresenta os resultados da notificação por classificação e evolução. Chama atenção que $5315(44,6 \%)$ dos casos suspeitos tenham sido encerrados sem a investigação dos mesmos. Isto mostra a péssima qualidade do banco, apresentando inconsistências quando as variáveis são associadas.
$\mathrm{NaTabela} 4$, foi realizado o cruzamento das variáveis do perfil sociodemográfico com as categorias "confirmados", "descartados" e "suspeitos" da variável classificação. Com exceção da variável sexo $(p=0,823)$, todas as demais obtiveram o $p$-valor menor igual a 0,001, indicando a não homogeneidade dos grupos estudados.

Observa-se uma desigualdade da variável raça/cor parda $(46,7 \%)$ e preta $(5,3 \%)$ como casos suspeitos, bem como analfabetos (12.1\%) neste grupo. Estes percentuais são maiores no grupo suspeito. Outra observação é encontrar um grande percentual de sintomas no grupo suspeito.

\section{DISCUSSÃO}

Os dados internacionais sugerem que as crianças com menos de 18 anos representam cerca de 8,5\% dos casos notificados, com relativamente poucas mortes em comparação com outras faixas etárias e geralmente apresentam quadro leve da doença ${ }^{11}$.

O estudo apontou que os dados disponíveis dos casos registrados de até 19 anos de idade no painel eletrônico para a COVID-19 no Estado do Espírito Santo apresentaram classificação entre ruim e regular para muitas variáveis necessárias para o planejamento de ações e tomada de decisões em saúde pública para o controle da doença. Destaca-se qualidade regular para o critério de confirmação e raça/cor da pele, qualidade ruim para a classificação do caso, status da notificação e escolaridade, e qualidade muito ruim para a evolução do caso. Soma-se a isso, o fato de muitos casos classificados como suspeitos não terem informações sobre a evolução do caso. Ademais, ao realizar análise comparando variáveis do perfil epidemiológico das crianças, adolescentes e jovens com as categorias "confirmados", "descartados" "e "suspeitos" da variável classificação, observouse a não homogeneidade dos grupos estudados.

As crianças infectadas pelo SARSCoV-2, diferentemente dos adultos, apresentam formas clínicas leves ou assintomáticas na maior parte dos casos descritos na literatura. Provavelmente, a condição de portadora assintomática possui grande impacto nas formas de transmissão do vírus na comunidade, já que a identificação de portadores assintomáticos é um grande desafio em todo o mundo ${ }^{12}$. É de extrema importância que os dados referentes a essa faixa etária sejam preenchidos corretamente e os casos suspeitos sejam concluídos, para que se possa identificar o mais precocemente os casos positivos e identificar o perfil dessa população que está contaminada, e, dessa forma, adotar medidas preventivas para diminuir a circulação viral, evitando a propagação da doença.

Estudos internacionais e brasileiros têm apontado à questão social da pandemia da COVID-19, difundindo-se das áreas mais ricas das grandes cidades para as mais pobres com baixa escolaridade e majoritariamente de indivíduos negros ${ }^{13-17}$. Estas variáveis, escolaridade e raça/cor da pele, permitem traçar o perfil epidemiológico dos indivíduos afetados pela pandemia e pensar em estratégias no campo da saúde, atreladas às ações sociais que possam mitigar o evento sobre o público mais afetado ${ }^{18}$. Todavia, assim como observado em estudos sobre a epidemiologia da COVID-19 ou outras doenças infecciosas de importância para 
Tabela 1. Incompletude das variáveis dos indivíduos de 0 a 19 anos notificados e disponibilizadas no painel eletrônico da COVID-19 no Espírito Santo.

\begin{tabular}{|c|c|c|c|c|}
\hline Variável & Categoria & $\mathrm{N}$ & $\%$ & Classificação \\
\hline Classificação & Suspeito & 12062 & $44 \%$ & RU \\
\hline Evolução & Em branco & 21725 & $79 \%$ & MR \\
\hline Critério Confirmação & Em branco & 4334 & $16 \%$ & $\mathrm{R}$ \\
\hline Status Notificação & Em Aberto & 8267 & $30 \%$ & $\mathrm{RU}$ \\
\hline Bairro & Em branco & 82 & $0 \%$ & $E$ \\
\hline Sexo & Ignorado & 5 & $0 \%$ & $\mathrm{E}$ \\
\hline Raça/Cor & Ignorado & 4758 & $17 \%$ & $\mathrm{R}$ \\
\hline Escolaridade & Ignorado & 6959 & $25 \%$ & RU \\
\hline Febre & Em branco & 77 & $0 \%$ & $\mathrm{E}$ \\
\hline Dificuldade Respiratória & Em branco & 74 & $0 \%$ & $\mathrm{E}$ \\
\hline Tosse & Em branco & 73 & $0 \%$ & E \\
\hline Coriza & Em branco & 71 & $0 \%$ & $\mathrm{E}$ \\
\hline Dor de Garganta & Em branco & 100 & $0 \%$ & $\mathrm{E}$ \\
\hline Diarreia & Em branco & 71 & $0 \%$ & $E$ \\
\hline Cefaleia & Em branco & 95 & $0 \%$ & E \\
\hline Comorbidade Pulmão & Em branco & 93 & $0 \%$ & $\mathrm{E}$ \\
\hline Comorbidade Cardiovascular & Em branco & 87 & $0 \%$ & $\mathrm{E}$ \\
\hline Comorbidade Renal & Em branco & 87 & $0 \%$ & $\mathrm{E}$ \\
\hline Comorbidade Diabetes & Em branco & 89 & $0 \%$ & $\mathrm{E}$ \\
\hline Comorbidade Tabagismo & Em branco & 89 & $0 \%$ & $\mathrm{E}$ \\
\hline Comorbidade Obesidade & Em branco & 98 & $0 \%$ & $\mathrm{E}$ \\
\hline \multirow{2}{*}{ Ficou Internado } & Ignorado & 312 & $1 \%$ & $\mathrm{E}$ \\
\hline & Não Informado & 11998 & $44 \%$ & $\mathrm{RU}$ \\
\hline \multirow{2}{*}{ Viagem Brasil } & Ignorado & 502 & $2 \%$ & $E$ \\
\hline & Não Informado & 7768 & $28 \%$ & RU \\
\hline \multirow{2}{*}{ Viagem Internacional } & Ignorado & 402 & $1 \%$ & $\mathrm{E}$ \\
\hline & Não Informado & 10751 & $39 \%$ & $\mathrm{RU}$ \\
\hline \multirow{2}{*}{ Profissional Saúde } & Ignorado & 1603 & $6 \%$ & B \\
\hline & Não Informado & 199 & $1 \%$ & $\mathrm{E}$ \\
\hline Possui Deficiência & Não Informado & 2869 & $10 \%$ & B \\
\hline Morador De Rua & Não Informado & 3105 & $11 \%$ & $\mathrm{R}$ \\
\hline
\end{tabular}

Fonte: Painel COVID do ES, 2020.

$E=$ excelente, $B=$ bom, $R=$ regular, $R U=$ ruim, $M R=$ muito ruim

a saúde pública no Brasil, as variáveis escolaridade e raça/cor da pele não possuem boa completude ${ }^{13-17}$. Em relação à variável raça/cor da pele, estudo realizado com dados de internação por COVID-19 no Brasil demonstrou apenas $62 \%$ de completude desta variável ${ }^{13}$. Aliás, deve-se destacar o protagonismo do movimento negro na inserção desta variável na ficha de notificação da COVID-19, pois embora a Portaria no 992 de 13 de maio de 2009, que institui a Política Nacional de Saúde Integral da População
Negra (PNSIPN) e a Portaria ํㅜ 344 de $1^{\circ}$ de fevereiro de 2017 , que dispõe sobre o preenchimento do quesito raça/cor nos formulários dos sistemas de informação em saúde, no início da epidemia no Brasil esta variável não estava disponível na ficha de notificação de doenças por COVID-19, sendo incluída e estabelecida como obrigatória após ações do movimento negro ${ }^{19,20}$.

Evidenciou-se que a proporção de informação ignorada e deixada em branco foi elevada para as variáveis de conclusão 
Tabela 2. Frequência do resultado da classificação versus evolução e critério de confirmação das notificações por COVID -19 de indivíduos de 0 a 19 anos no Espírito Santo, 2020.

\begin{tabular}{|c|c|c|c|c|c|c|c|c|c|}
\hline \multirow{3}{*}{ Classificação } & \multirow{3}{*}{ Evolução } & \multicolumn{8}{|c|}{ Critério Confirmação } \\
\hline & & \multicolumn{2}{|c|}{ Sem Informação } & \multicolumn{2}{|c|}{ Clínico } & \multicolumn{2}{|c|}{$\begin{array}{c}\text { Clínico } \\
\text { Epidemiológico }\end{array}$} & \multicolumn{2}{|c|}{ Laboratorial } \\
\hline & & $\mathrm{N}$ & $\%$ & $\mathrm{~N}$ & $\%$ & $\mathrm{~N}$ & $\%$ & $\mathrm{~N}$ & $\%$ \\
\hline \multirow{3}{*}{ Confirmados } & Cura & 42 &, $8 \%$ & 177 & $3,3 \%$ & 852 & $15,8 \%$ & 4333 & $80,2 \%$ \\
\hline & Em branco & 61 & $9,0 \%$ & 6 & $0,9 \%$ & 45 & $6,6 \%$ & 566 & $83,5 \%$ \\
\hline & Óbito pelo COVID19 & 0 & $0,0 \%$ & 0 & $0,0 \%$ & 0 & $0,0 \%$ & 17 & $100,0 \%$ \\
\hline \multirow{3}{*}{ Descartados } & Cura & 2 & $14,3 \%$ & 0 & $0,0 \%$ & 3 & $21,4 \%$ & 9 & $64,3 \%$ \\
\hline & Em branco & 271 & $3,0 \%$ & 266 & $2,9 \%$ & 466 & $5,1 \%$ & 8133 & $89,0 \%$ \\
\hline & Óbito por outras causas & 0 & $0,0 \%$ & 1 & $2,5 \%$ & 0 & $0,0 \%$ & 39 & $97,5 \%$ \\
\hline \multirow{2}{*}{ Suspeito } & Cura & 8 & $5,3 \%$ & 86 & $57,0 \%$ & 49 & $32,5 \%$ & 8 & $5,3 \%$ \\
\hline & Em branco & 3950 & $33,2 \%$ & 6317 & $53,0 \%$ & 1343 & $11,3 \%$ & 301 & $2,5 \%$ \\
\hline
\end{tabular}

Fonte: Painel COVID do ES, 2020.

Tabela 3. Frequências entre a classificação e o status da notificação de indivíduos de 0 a 19 anos disponibilizadas no painel eletrônico da COVID-19 no Espírito Santo, 2020.

\begin{tabular}{|c|c|c|c|c|c|}
\hline \multirow{3}{*}{ Classificação } & \multirow{3}{*}{ Evolução } & \multicolumn{4}{|c|}{ Status Notificação } \\
\hline & & \multicolumn{2}{|c|}{ Em Aberto } & \multicolumn{2}{|c|}{ Encerrado } \\
\hline & & $\mathrm{N}$ & $\%$ & $\mathrm{~N}$ & $\%$ \\
\hline \multirow{3}{*}{ Confirmados } & Cura & 24 & $0,4 \%$ & 5380 & $99,6 \%$ \\
\hline & Em branco & 595 & $87,8 \%$ & 83 & $12,2 \%$ \\
\hline & Óbito pelo COVID19 & 0 & $0,0 \%$ & 17 & $100,0 \%$ \\
\hline \multirow{3}{*}{ Descartados } & Cura & 3 & $21,4 \%$ & 11 & $78,6 \%$ \\
\hline & Em branco & 1032 & $11,3 \%$ & 8104 & $88,7 \%$ \\
\hline & Óbito por outras causas & 1 & $2,5 \%$ & 39 & $97,5 \%$ \\
\hline \multirow{2}{*}{ Suspeito } & Cura & 16 & $10,6 \%$ & 135 & $89,4 \%$ \\
\hline & Em branco & 6596 & $55,4 \%$ & 5315 & $44,6 \%$ \\
\hline
\end{tabular}

Fonte: Painel COVID do ES, 2020.

dos casos por COVID-19, implicando classificação da completude entre ruim e regular. $\mathrm{O}$ critério de confirmação apresentou completude regular, e a classificação do caso e status da notificação com preenchimento ruim. Observou- se também qualidade muito ruim para a evolução do caso. Soma-se a isso, o fato de muitos casos classificados como suspeitos não terem informações sobre a evolução do caso. A presença de casos inconclusivos, para os quais não foi possível descartar ou confirmar o adoecimento, pode ocorrer devido a dados insuficientes, a campos da ficha deixados em branco ou preenchidos de forma inadequada, ou ainda, por conta da não coleta de amostras para a realização dos exames diagnósticos ${ }^{2}$. Essas variáveis são de extrema importância para o encerramento do caso $e$ para uma atuação mais ativa da vigilância epidemiológica. Uma vez que tais dados não são coletados no primeiro momento da investigação, faz-se necessário seu acompanhamento para posterior preenchimento dessas variáveis nas fichas de notificação dos agravos em saúde ${ }^{2}$.

O encerramento dos casos notificados de um agravo à saúde em tempo hábil permite o conhecimento dos casos confirmados e descartados, e a adoção de medidas de controle em tempo oportuno pela vigilância epidemiológica, principalmente em uma pandemia ocasionada por um vírus novo. O sub-registro dos casos pode ser atribuído a diversos fatores, como atraso nas notificações, digitação dos dados, problemas no processamento e transferência das informações, assim como à demora na liberação dos resultados de exames, ausência de atualização dos dados ou de uma retroalimentação adequada da fonte notificadora, o que também pode gerar desestímulo e descontinuidade do processo de investigação e encerramento do caso ${ }^{2,21,22}$. 
Tabela 4. Associação entre as variáveis do estudo e a classificação do caso das notificações por COVID -19 de indivíduos de 0 a 19 anos no Espírito Santo, 2020.

\begin{tabular}{|c|c|c|c|c|c|c|c|}
\hline \multirow{3}{*}{ Variável } & \multirow{3}{*}{ Categoria } & \multicolumn{6}{|c|}{ Classificação } \\
\hline & & \multicolumn{2}{|c|}{ Confirmados } & \multicolumn{2}{|c|}{ Descartados } & \multicolumn{2}{|c|}{ Suspeito } \\
\hline & & $\mathrm{N}$ & $\%$ & $\mathrm{~N}$ & $\%$ & $\mathrm{~N}$ & $\%$ \\
\hline \multirow{2}{*}{ Sexo } & $\mathrm{F}$ & 3188 & $52,3 \%$ & 4769 & $51,9 \%$ & 6318 & $52,4 \%$ \\
\hline & M & 2909 & $47,7 \%$ & 4420 & $48,1 \%$ & 5742 & $47,6 \%$ \\
\hline \multirow{6}{*}{ Raça/Cor } & Amarela & 439 & $7,2 \%$ & 509 & $5,5 \%$ & 925 & $7,7 \%$ \\
\hline & Branca & 2141 & $35,1 \%$ & 2956 & $32,2 \%$ & 3089 & $25,6 \%$ \\
\hline & Ignorado & 1161 & $19,0 \%$ & 1830 & $19,9 \%$ & 1767 & $14,6 \%$ \\
\hline & Indígena & 18 & ,3\% & 28 & ,3\% & 11 & ,1\% \\
\hline & Parda & 2114 & $34,7 \%$ & 3463 & $37,7 \%$ & 5630 & $46,7 \%$ \\
\hline & Preta & 226 & $3,7 \%$ & 404 & $4,4 \%$ & 640 & $5,3 \%$ \\
\hline \multirow{11}{*}{ Escolaridade } & Analfabeto & 449 & $7,4 \%$ & 910 & $9,9 \%$ & 1454 & $12,1 \%$ \\
\hline & 1a a 4a série incompleta do EF & 502 & $8,2 \%$ & 843 & $9,2 \%$ & 1175 & $9,7 \%$ \\
\hline & 4a série completa do EF & 151 & $2,5 \%$ & 197 & $2,1 \%$ & 294 & $2,4 \%$ \\
\hline & 5 a à 8 a série incompleta do EF & 776 & $12,7 \%$ & 1045 & $11,4 \%$ & 1413 & $11,7 \%$ \\
\hline & Ensino fundamental completo & 306 & $5,0 \%$ & 475 & $5,2 \%$ & 604 & $5,0 \%$ \\
\hline & Ensino médio incompleto & 779 & $12,8 \%$ & 997 & $10,8 \%$ & 1345 & $11,2 \%$ \\
\hline & Ensino médio completo & 746 & $12,2 \%$ & 1060 & $11,5 \%$ & 1220 & $10,1 \%$ \\
\hline & Educação superior incompleta & 151 & $2,5 \%$ & 216 & $2,4 \%$ & 190 & $1,6 \%$ \\
\hline & Educação superior completa & 48 &, $8 \%$ & 84 &, $9 \%$ & 67 &, $6 \%$ \\
\hline & Ignorado & 1683 & $27,6 \%$ & 2319 & $25,2 \%$ & 2957 & $24,5 \%$ \\
\hline & Não se aplica & 508 & $8,3 \%$ & 1044 & $11,4 \%$ & 1343 & $11,1 \%$ \\
\hline \multirow{4}{*}{ Faixa Etária } & 0 a 4 anos & 1212 & $19,9 \%$ & 2410 & $26,2 \%$ & 3531 & $29,3 \%$ \\
\hline & 5 a 9 anos & 879 & $14,4 \%$ & 1516 & $16,5 \%$ & 1903 & $15,8 \%$ \\
\hline & 10 a 14 anos & 1219 & $20,0 \%$ & 1542 & $16,8 \%$ & 1936 & $16,1 \%$ \\
\hline & 15 a 19 anos & 2789 & $45,7 \%$ & 3722 & $40,5 \%$ & 4692 & $38,9 \%$ \\
\hline Febre & Sim & 2817 & $46,6 \%$ & 4164 & $45,4 \%$ & 7060 & $58,6 \%$ \\
\hline Dificuldade Respiratória & Sim & 834 & $13,8 \%$ & 1905 & $20,8 \%$ & 2253 & $18,7 \%$ \\
\hline Tosse & Sim & 2572 & $42,6 \%$ & 4542 & $49,5 \%$ & 7272 & $60,3 \%$ \\
\hline Coriza & Sim & 2220 & $36,7 \%$ & 3693 & $40,2 \%$ & 6143 & $50,9 \%$ \\
\hline Dor de Garganta & Sim & 1502 & $24,9 \%$ & 2475 & $27,0 \%$ & 4087 & $34,0 \%$ \\
\hline Diarreia & Sim & 791 & $13,1 \%$ & 1307 & $14,2 \%$ & 1545 & $12,8 \%$ \\
\hline Cefaleia & Sim & 2444 & $40,4 \%$ & 3192 & $34,8 \%$ & 5117 & $42,5 \%$ \\
\hline Comorbidade Pulmão & Sim & 402 & $6,7 \%$ & 910 & $9,9 \%$ & 1184 & $9,8 \%$ \\
\hline Comorbidade Cardio & Sim & 63 & $1,0 \%$ & 115 & $1,3 \%$ & 95 & ,8\% \\
\hline Comorbidade Renal & Sim & 17 & ,3\% & 23 & ,3\% & 9 & , 1\% \\
\hline Comorbidade Diabetes & Sim & 28 &, $5 \%$ & 36 & , $4 \%$ & 26 &, $2 \%$ \\
\hline Comorbidade Tabagismo & Sim & 23 &, $4 \%$ & 40 & ,4\% & 104 & ,9\% \\
\hline Comorbidade Obesidade & Sim & 81 & $1,3 \%$ & 132 & $1,4 \%$ & 108 & ,9\% \\
\hline Ficou Internado & Sim & 50 &, $8 \%$ & 173 & $1,9 \%$ & 46 &, $4 \%$ \\
\hline Viagem Brasil & Sim & 230 & $3,8 \%$ & 396 & $4,3 \%$ & 382 & $3,2 \%$ \\
\hline Viagem Internacional & Sim & 2 &, $0 \%$ & 31 &, $3 \%$ & 3 &, $0 \%$ \\
\hline Profissional Saúde & Sim & 97 & $1,6 \%$ & 210 & $2,3 \%$ & 144 & $1,2 \%$ \\
\hline Possui Deficiência & Sim & 252 & $4,1 \%$ & 269 & $2,9 \%$ & 374 & $3,1 \%$ \\
\hline Morador de Rua & Sim & 28 &, $5 \%$ & 38 &, $4 \%$ & 61 &, $5 \%$ \\
\hline
\end{tabular}

Fonte: Painel COVID do ES, 2020. 


\section{CONCLUSÃO E IMPLICAÇÕES PARA A PRÁTICA}

Os resultados encontrados na avaliação da qualidade dos dados do Painel COVID ES demonstram completude ruim a regular para diversas variáveis do estudo. As análises realizadas a partir de dados de má qualidade podem não representar o real perfil epidemiológico dos agravos, prejudicando a adoção de medidas de controle mais adequadas e a identificação das regiões de risco ${ }^{2,23}$. Dessa forma, evidencia-se a necessidade de se adotar uma avaliação sistemática da qualidade da informação gerada pelos sistemas de informações em saúde.

Embora o estudo revele achados importantes para a vigilância e avaliação da qualidade da informação em saúde considerando a disponibilidade dos dados abertos em tempo real, o mesmo apresenta uma limitação que reside, justamente, em suas características regionais, específicas ao Espírito Santo, não extensivas à realidade de outros estados do país, uma vez que cada estado adotou modos informativos operantes diferentes neste contexto de pandemia por COVID-19, não ocorrendo uma padronização da disponibilidade dos dados em nível federal.

Deve-se levar em consideração que no início da pandemia da COVID-19 o Brasil realizava as notificações de casos suspeitos através de formulário on-line chamado FormSus, no qual somente os gestores de saúde dos estados possuem acesso às informações. Contudo, no dia 29 de março o estado do Espírito Santo passou a realizar as notificações através do aplicativo on-line e-SUS VS ${ }^{24}$. Esse aplicativo desenvolvido pela Secretaria Estadual de Saúde do Espírito Santo fornece informações em tempo real a todas as esferas de gestão e saúde, através do preenchimento e da edição on-line dos formulários, no que capilariza às notificações a todas unidades de assistência em saúde e traz clareza e rapidez ao dado. Todavia, é necessário educação permanente dos profissionais de saúde, treinar os mesmos para o preenchimento dos dados no e-SUS de forma correta, evitando deixar campos em branco ou preenchê-los como 'Ignorado', e conscientizá-los que através do perfil epidemiológico serão verificados os grupos de risco, as populações mais vulneráveis para adoecer, e tratando-se de uma pandemia por um vírus novo, esses dados devem estar disponíveis imediatamente, e com qualidade para que medidas de prevenção e estratégias de controle da doença possam ser implementadas pelos serviços e órgãos públicos.

\section{CONTRIBUIÇÕES DOS AUTORES}

Desenho do estudo. Ethel Leonor Maciel. Eliana Zandonade. Thiago Nascimento do Prado

Extração dos dados. Eliana Zandonade. Etereldes Goncalves Jr. Análise de dados. Ethel Leonor Maciel. Etereldes Goncalves Jr. Eliana Zandonade.

Interpretação dos resultados. Ethel Leonor Maciel. Etereldes Goncalves Jr. Priscila Carminati Siqueira. Thiago Nascimento do Prado. Eliana Zandonade.
Redação e revisão crítica do manuscrito. Ethel Leonor Maciel. Pablo Medeiros Jabor. Etereldes Goncalves Jr. Priscila Carminati Siqueira. Thiago Nascimento do Prado. Eliana Zandonade.

Aprovação da versão final do artigo. Ethel Leonor Maciel. Pablo Medeiros Jabor. Etereldes Goncalves Jr. Priscila Carminati Siqueira. Thiago Nascimento do Prado. Eliana Zandonade.

Responsabilidade por todos os aspectos do conteúdo e a integridade do artigo publicado. Ethel Leonor Maciel. Pablo Medeiros Jabor. Etereldes Goncalves Jr. Priscila Carminati Siqueira. Thiago Nascimento do Prado. Eliana Zandonade.

\section{EDITOR ASSOCIADO}

Gerson Luiz Marinho (D)

\section{SCIENTIFIC EDITOR}

\author{
Ivone Evangelista Cabral (1)
}

\section{REFERÊNCIAS}

1. Portaria n. 264 de 17 de fevereiro de 2020 (BR). Altera a Portaria de Consolidação n. 4/GM/MS, de 28 de setembro de 2017. Diário Oficial da União [periódico na internet], Brasília (DF), 19 fev 2020 [citado 13 fev 2021]. Disponível em: http://www.in.gov.br/en/web/dou/-/portarian-264-de-17-de-fevereiro-de-2020-244043656

2. Siqueira PC, Maciel ELN, Catão RC, Brioschi AP, Silva TCC, Prado TN Completude das fichas de notificação de febre amarela no estado do Espírito Santo, 2017. Epidemiol Serv Saude. 2020;29(3):e2019402. http://dx.doi.org/10.5123/S1679-49742020000300014.PMid:32555932.

3. Klaucke DN, Buehler JW, Thacker SB, Parrish G, Trowbridge FL, Berkelman RL, Surveillance Coordination Group. Guidelines for evaluating surveillance systems: recommendations from the guidelines working group. Morb Mortal Wkly Rep [Internet]. 1988 May [citado 2020 ago 18];37(Suppl 5):1-18. Available from: https://www.cdc.gov/mmwr/ preview/mmwrhtml/00001769.htm

4. Braz RM, Tauil PL, Santelli ACFS, Fontes CJF. Avaliação da completude e da oportunidade das notificações de malária na Amazônia Brasileira, 2003-2012. Epidemiol Serv Saude. 2016;25(1):21-32. PMid:27861675.

5. Ministério da Saúde (BR). Norma operacional da assistência à saúde do Sistema Único de Saúde. NOAS - SUS. Brasília: Ministério da Saúde; 2002.

6. Espírito Santo. Governo do Estado. Painel Covid-19 - Estado do Espírito Santo [Internet]. [citado 20 ago 2020]. 2020. Disponível em: https:// coronavirus.es.gov.br/painel-covid-19-es

7. Espírito Santo. Governo do Estado. Secretaria Estadual de Saúde. NOTA TÉCNICA COVID-19 N. 073/2020 - GEVS/SESA/ES [Internet] Viória (ES): Secretaria Estadual de Saúde; 2020 [citado 2021 fev 10] Disponível em: coronavirus.es.gov.br>Coronavirus>NotasTecnicas

8. Romero DE, Cunha AB. Avaliação da qualidade das variáveis sócioeconômicas e demográficas dos óbitos de crianças menores de um ano registrados no Sistema de Informação Sobre Mortalidade do Brasil (1996/2001). Cad Saude Publica. 2006;22(3):673-84. http://dx.doi. org/10.1590/S0102-311X2006000300022. PMid:16583111.

9. Comisión Econômica para América Latina y el Caribe. Gestión orientada a asegurar la calidad de los dados en los institutos nacionales de Estadística. Santiago de Chile: Comisión Económica para América Latina y el Caribe; 2003.

10. Felix JD. Padrões espaço-temporais da mortalidade por câncer de mama em mulheres no Estado do Espírito Santo - um estudo ecológico [dissertação]. Vitória: Universidade Federal do Espírito Santo- UFES; 2011.

11. World Health Organization. Coronavirus disease (COVID 19): schools [Internet]. Genebra: WHO; 2020 [citado 2021 fev 13]. Disponível em: 
https://www.who.int/news-room/q-a-detail/coronavirus-disease-covid19-schools

12. Martins MM, Prata-Barbosa A, Magalhães-Barbosa MCD, Cunha AJLA Características clínicas e laboratoriais da infecção por sars-cov-2 em crianças e adolescentes. Rev. paul. pediatra. [Internet]. 2021 [citado 2021 fev 18];39:e2020231. Disponível em: http://www.scielo.br/scielo. php?script=sci_arttext\&pid=S0103-05822021000100507\&lng=en

13. Baqui P, Bica I, Marra V, Ercole A, van der Schaar M. Variações étnicas e regionais na mortalidade hospitalar por COVID-19 no Brasil: um estudo transversal observacional. Lancet. 2020;8(8):e1018-26.

14. Cavalcante JR, Abreu ADJL. COVID-19 in the city of Rio de Janeiro: spatial analysis of first confirmed cases and deaths. Epidemiol Serv Saude. 2020;29(3):e2020204. http://dx.doi.org/10.5123/s167949742020000300007. PMid:32520107.

15. Krouse HJ. COVID-19 and the Widening Gap in Health Inequity Otolaryngol Head Neck Surg. 2020;163(1):65-6. http://dx.doi. org/10.1177/0194599820926463. PMid:32366172.

16. Goes EF, Ramos DDO, Ferreira AJF. Desigualdades raciais em saúde e a pandemia da Covid-19. Trab Educ Saúde. 2020;18(3):e00278110. http://dx.doi.org/10.1590/1981-7746-sol00278.

17. Millett GA, Jones AT, Benkeser D, Baral S, Mercer L, Beyrer C et al. Assessing differential impacts of COVID-19 on black communities. Ann Epidemiol. 2020;47:37-44. http://dx.doi.org/10.1016/j.annepidem.2020.05.003. PMid:32419766.

18. Santos HLPC, Maciel FBM, Santos KR, Conceição CDVS, Oliveira RS, Silva NRF, et al. Necropolítica e reflexões acerca da população negra no contexto da pandemia da COVID-19 no Brasil: uma revisão bibliográfica. Ciênc. saúde coletiva. 2020;25(Suppl 2):4211-24. https:// doi.org/10.1590/1413-812320202510.2.25482020.

19. Portaria ${ }^{\circ}$ 992, de 13 de maio de 2009 (BR). Institui a Política Nacional de Saúde Integral da População Negra (PNSIPN). Diário Oficial da União, Brasília (DF); 2009.

20. Portaria $n^{\circ} 344$, de $1^{\circ}$ de fevereiro de 2017 (BR). Dispõe sobre o preenchimento do quesito raça/cor nos formulários dos sistemas de informação em saúde. Diário Oficial da União, Brasília (DF); 2017.

21. Silva GDM, Bartholomay P, Cruz OG, Garcia LP. Avaliação da qualidade dos dados, oportunidade e aceitabilidade da vigilância da tuberculose nas microregiões do Brasil. Cien Saude Colet. 2017;22(10):3307-19. http:// dx.doi.org/10.1590/1413-812320172210.18032017. PMid:29069186.

22. Meirelles MQB, Lopes AKB, Lima KC. Vigilância epidemiológica de HIV/Aids em gestantes: uma avaliação acerca da qualidade da informação disponível. Rev Panam Salud Publica. 2016;40(6):327-34. PMid:28718491.

23. German RR, Westmoreland D, Armstrong G, Birkhead GS, Horan JM, Center for Diseases Control and Prevention, et al. Updated guidelines for evaluating public health surveillance systems: recommendations from the guidelines working group. Morb Mortal Wkly Rep. [Internet]. 2001 Jul [citado 2020 maio 8];50(RR13):1-35. Disponível em: https:/ www.cdc.gov/mmwr/preview/mmwrhtml/rr5013a1.htm

24. Ministério da Saúde (BR). DATASUS [Internet]. 2020 [citado 2020 ago 23]. Disponível em: https://www.saude.ce.gov.br/2020/03/26/ datasus-disponibiliza-nova-plataforma-para-notificacao-de-casosde-covid-19/\&sa=D\&ust=1602420619162000\&usg=AFQjCNFgo8itmWCqKdweQNSjblpCUdeog 